\title{
Critérios de relevância e classificação política no discurso jornalístico internacional
}

\author{
Fernando Ewerton Fernandez Júnior ${ }^{I}$ \\ https://orcid.org/0000-0001-6437-7112
}

\section{Rosali Fernandez de Souza ${ }^{I I}$}

https://orcid.org/0000-0002-0890-7999

${ }^{I}$ Universidade Federal do Rio de Janeiro, RJ, Brasil.

Professor Adjunto do Departamento de Expressão e Linguagens

da Escola de Comunicação da UFRJ.

II Instituto Brasileiro de Informação em Ciência da Informação e Tecnologia, RJ, Brasil.

Doutora em Ciência da Informação. Docente do Programa de Pós-Graduação em Ciência da Informação do IBICT.

\section{http://dx.doi.org/10.1590/1981-5344/3941}

Na era do conteúdo indexado por ferramentas de busca na internet, agências de notícia transnacionais e veículos jornalísticos tradicionais em outras mídias continuam sendo importantes fornecedores de informação para bases de dados nos principais idiomas do planeta, seguindo seus próprios critérios de relevância na escolha do que, e como, informar. A fim de mapear as classificações políticas adotadas na cobertura da eleição presidencial brasileira de 2010, este artigo analisa termos presentes antes, depois ou em substituição ao nome dos quatro principais candidatos - Dilma Rousseff $(P T)$, José Serra (PSDB), Marina Silva (PV) e Plínio de Arruda Sampaio (PSOL) - em 261 textos noticiosos, em inglês e 
espanhol, de quatro agências (AFP, AP, EFE e Reuters) e quatro veículos (as emissoras de TV BBC e CNN, e os jornais El País e The New York Times). A partir dos resultados, são traçados paralelos entre os critérios adotados pelas fontes jornalísticas ao atribuírem relevância às informações associadas a cada candidato e as generalizações de Tefko Saracevic (2009) sobre como usuários de sistemas de informação inferem relevância em processos de busca, sugeridas com base em 40 anos de estudos em Ciência da Informação.

Palavras-chave: relevância; jornalismo; classificação política; eleições presidenciais.

\section{Criteria of relevanceandpolitical classificationin the internationaljournalistic discourse}

In an age in which content is indexed by Internet search tools, transnational new agencies and traditional news media are still important sources of information for data bases in the main languages, according to their own criteria of relevance on what and how to inform. With the purpose of mapping the political classifications adopted on the coverage of the 2010 Brazilian presidential election, this article analyses terms used before, after or as a substitution to the name of the four main candidates Dilma Rousseff (PT), José Serra (PSDB), Marina Silva, and Plínio de Arruda Sampaio (PSOL) -, present in 261 news texts, both in English and Spanish, of four agencies (AFP, $A P, E F E$ and Reuters) and four traditional news media (BBC and CNN TV networks, plus the newspapers El País and The New York Times). From these results, parallels are traced among the criteria adopted by the journalistic sources when attributing relevance to information associated to each candidate and the generalizations of Tefko Saracevic (2009) about how information system's users infer relevance in search processing, suggested 
based on 40 years of studies in the field of Information Science.

Keywords: relevance; journalism; classification; politics; elections.

Recebido em 08.04.2019. Aceito em 29.06.2020

\section{Introdução}

$\mathrm{Na}$ era do conteúdo indexado por ferramentas de busca na internet, agências de notícia com origem no século XIX e veículos jornalísticos tradicionais em outras mídias continuam sendo importantes fornecedores de informação para bases de dados nos principais idiomas do planeta, distribuindo conceitos e representações de fatos ocorridos em todo e qualquer lugar do mundo, segundo seus próprios critérios de relevância na escolha do que, e como, informar.

Assim como revolucionou a forma de recebermos informação, a internet vem redefinindo também o modo como as notícias são produzidas, apoiadas em estratégias de indexação para ganhar visibilidade nos portais agregadores e nas ferramentas de busca. Os resultados são conteúdos cada vez mais parecidos entre si, replicados em vários sites, mas procedentes de poucas fontes, como demonstram as pesquisas de Paterson (2006) e do Project for Excellence in Journalism(OLMSTEAD; MITCHELL; ROSENSTIEL, 2011).

Mesmo entre fontes de mídias, idiomas e culturas diferentes, as variações muitas vezes são sutis, mas significativas, como demonstra a análise de conteúdo das notícias sobre a eleição presidencial brasileira de 2010, distribuídas em inglês e espanhol na internet por quatro agências transnacionais - a francesa France Press e (AFP), a americana Associated Press (AP), a espanhola EFE e a canadense de origem britânica Reuters e quatro veículos jornalísticos tradicionais - os jornais The New York Times (NYT), dos Estados Unidos, e El País, da Espanha, e as emissoras de TV British Broadcast Corporation (BBC), britânica, e Cable News Network (CNN), americana.

Para identificaros critérios de relevância aplicados em diferentes fontes e idiomas, foram analisados neste estudo 261 textos, coletados em torno do $1^{0}$ e do $2^{\circ}$ turnos da eleição de 2010, e mapeados termos presentes antes, depois ou em substituição ao nome dos quatro principais candidatos: Dilma Rousseff (Partido dos Trabalhadores, PT), José Serra (Partido da Social Democracia Brasileira, PSDB), Marina Silva (Partido 
Verde, PV) e Plínio de Arruda Sampaio (Partido Socialismo e Liberdade, PSOL).

A partir dos resultados, foram traçados paralelos entre os critérios adotados pelas fontes ao atribuírem relevância às informações associadas a cada candidato e as generalizações de Saracevic (2009) sobre como usuários de sistemas de informação inferem relevância em processos de busca, sugeridas com base em 40 anos de estudos em Ciência da Informação. Buscou-se, assim, identificar fatores organizacionais, idiomáticos e culturais presentes nas representações políticas que circulam em sites jornalísticos de escala mundial, com impacto nos resultados que os usuários de ferramentas de busca podem encontrar na internet.

\section{Referencial teórico}

Indagações sobre o que faz uma informação relevante, e para quem, permeiam diversos estudos em Ciência da Informação. Dentre os muitos pesquisadores do tema, Pinheiro (2004) destaca a contribuição teórica de Tefko Saracevic à construção do conceito de relevância, fundamental na comunicação entre indivíduos e destes com os sistemas de informação. Na segunda parte de sua revisão do conceito, 30 anos após a primeira, de 1975, Saracevic (2009, p.71) considera que "relevância é uma, senão mesmo a noção chave em ciência da informação em geral e em recuperação de informação em particular", definindo-a como uma noção humana, não de sistema, resultado da interação entre usuários e sistemas na recuperação de informação.

Numerosos autores examinaram uma variedade de fatores que afetam como os humanos determinam relevância de uma informação ou de objetos de informação. Ao propor uma "visão epistemológica" "subject know ledge view" -, Hjørland(2010, p.217; 219) prega que "o estudo avançadoda recuperação da informaçãodevebasear-se noestudo das propriedadesda literaturae sua representaçãona terminologia do assunto,que é novamenterepresentadaem bases de dados".

Um aspecto importantedas bases de dados, ubiquamente acessíveis através das ferramentas de busca na internet, éque os termossão tomados fora de contextoe, portanto,partede seu significado é perdida. Tais situações, segundo Orrico e Oliveira (2006, p.143), "potencializam as dificuldades nos processos de transferência de informação, envolvendo trocas de conteúdos discursivos, principalmente porque ocorrem em contextos sociais muito distintos entre si".

Apesar de as mensagens serem abertas a diferentes interpretações, Aldé e Veiga (2004, p.501) ressaltam que "um texto possui uma leitura preferencial, aquela para a qual a própria mensagem procura atrair 0 
leitor" através do trabalho de codificação, no qual constrói os limites e parâmetros dentro dos quais a decodificação irá operar. Ao selecionar um assunto e a forma de abordá-lo, agências de notícias e veículos jornalísticos aplicam critérios de relevância com impacto nos conteúdos que os mecanismos de busca poderão encontrar.

Este estudo aborda um tema recorrente no campo da Comunicação, também presente em bases de dados objeto de pesquisas em Ciência da Informação: o discurso jornalístico, definido aqui como um tipo de texto caracterizado por um conjunto de regras narrativas de caráter sintático e semântico, com pretensão de validade enunciativa, adotado em geral por veículos noticiosos em diversos gêneros. Destes, o mais próximo de uma imagem prototípica são as notícias, entendidas como uma instância de texto narrativo que "envolve, em geral, vários personagens, mas não pode haver pressuposição sobre o conhecimento prévio do leitor com relação àqueles personagens, uma vez que a função/propósito desse gênero de discurso é informar" (PAREDES SILVA, 2007, p.171).

Adotamos, nesta análise, a perspectiva cognitivista que permeia os dois campos de estudo envolvidos, com foco na noção de enquadramento (ENTMAN, 1993; GITLIN, 1980) e no conceito de relevância, principal elo entre produção jornalística e sistemas de informação, dado o papel central que desempenha em ambos. Considerando que relevância é uma noção humana, que implica interação com o conhecimento representado em documentos armazenados em bases de dados, buscou-se identificarnas notícias coletadas termos indicadores dos frames (enquadramentos) adotados na construção do cenário de representação política (LIMA, 2004) pelo qual cada agência/veículo apresentou ao seu público/cliente (leitores, telespectadores, internautas) os candidatos a presidente do Brasil na eleição de 2010.

Entendemos que esse processo de codificação da "realidade" apoia-se em estruturas cognitivas que transcendem as visões instrumentalistas do jornalismo como espelho ou distorção da realidade, inspiradas na ideologia da objetividade (SCHUDSON, 1978; TRAQUINA, 2001), e vão além de questões puramente organizacionais - em que pesem os processos e as rotinas envolvidos na produção e distribuição de notícias. Na lógica operacional da atividade jornalística, a mensagem deve circular rapidamente e atingir a maior audiência no menor tempo possível, - que necessariamente implica numa padronização de linguagens e práticas, como as tipificações descritas por Tuchman (1973), entre outros.

A informação resultante carrega os elementos narrativos de sua elaboração, reflexos de modelos cognitivos (LAKOFF, 1987) que estruturam a formação de categorias organizadoras dos diversos "pacotes 
interpretativos" (GAMSON; MODIGLIANI, 1989) presentes nos textos jornalísticos, apoiados em classificações que servem como "tentativa de regularizar o movimento de informação de um contexto a outro" (BOWKER; STAR, 2000, p.290).

Para mapear alguns dos "pacotes" presentes em notícias sobre a eleição presidencial brasileira de 2010, este artigo concentra-se nas classificações políticas, entendidas como um conjunto estruturado de conceitos representados em termos específicos associados a candidatos e seus partidos durante períodos eleitorais.

Ao analisá-las, são traçados paralelos entre os critérios de atribuição de relevância aplicados por agências e veículos noticiosos e as generalizações sugeridas por Saracevic (2009) sobre os critérios adotados por usuários de sistemas de informação para inferir relevância em processos de busca.

\section{Abordagem metodológica}

O corpus analisado inclui 261 notícias de 13 serviços jornalísticos: sete em espanhol (AFP, AP, EFE, Reuters, BBC, CNN e El País) e seis em inglês (AFP, AP, Reuters, BBC, CNN e NYT). A análise de conteúdo lexical foi adotada para identificar a ocorrência de termos capazes de revelar esquemas de classificação política nas notícias sobre a eleição presidencial brasileira de 2010, relacionados aos quatro principais candidatos: Dilma Rousseff (PT), José Serra (PSDB), Marina Silva (PV) e Plínio de Arruda Sampaio (PSOL).

Os textos foram coletados em dois períodos de 10 dias em torno do 10 e do $2^{\circ}$ turnos da eleição presidencial de 2010, nas seções "Internacional/World" do Google News ${ }^{1}$ - nas versões Estados Unidos, España, U.K. (United Kingdom) e U.S. (United States). Destes, 187indicam autoria de alguma das quatro agências, em páginas próprias ou de terceiros, sendo 128 textos em espanhol (AFP, AP, EFE, Reuters) e 59 em inglês (AFP, AP e Reuters). Outros 74 foram encontrados em sites de veículos de origem nacional, idiomática ou cultural semelhante à de alguma das quatro agências (exceção da AFP): o jornal espanhol El País (mesma nacionalidade da EFE), a rede de televisão britânica BBC (mesma origem da Reuters), o jornal The New York Times (NYT) e a rede de TV CNN (americanos como a AP). Seguindo o mesmo procedimento adotado com as agências, foram analisados 48 textos em espanhol (BBC, CNN, El País) e 26 em inglês (BBC, CNN, NYT).

O estudo é baseado no registro de ocorrências de termos entendidos como "vocábulo ou grupo de vocábulos que corresponde a

\footnotetext{
${ }^{1}$ http://news.google.com
} 
uma unidade de significação ou de função, como elemento constitutivo para a inteligibilidade do que se enuncia" (CAMARA JR., 1985, p.232) presentes antes, depois ou em substituição ao nome de um ou mais candidatos em uma frase. Foram mapeados cinco tipos de termos, exaustivos e exclusivos - abrangem todos os termos encontrados, com cada termo associado a apenas um tipo - comuns a todas as fontes, repetindo procedimento de análise anterior sobre o tema (FERNANDEZ JÚNIOR, 2013):

- Termo Político- associado ao candidato e/ou ao seu partido: "deloficialistaPT".

- Termo Partidário - que associa o candidato ao nome do partido: "fromPSDB".

- Termo Pessoal- associado à pessoa do candidato, mas não ao partido: "de 62 años".

- Termo Funcional - associado a atividades, cargos e funções: "governor".

- Termo Relacional - em relação com outros candidatos e partidos: "rival".

Partindo da premissa de que "relevância é mensurável" (SARACEVIC, 2009, p.146), adotou-se o princípio metodológico de que "uma maior frequência lexical encontra-se hipoteticamente associada a um maior peso relativo e relevância conceituais dentro de um conjunto de termos" (BIOLCHINI, 2001, p.5), considerando que a frequência dos termos no corpus revela os critérios de relevância das fontes na representação dos candidatos, para mapear as matrizes de classificação política presentes em notícias sobre a eleição presidencial brasileira de 2010, em espanhol e inglês.

\section{Análise e interpretação dos resultados}

Para identificar relações entre as classificações políticas adotadas por diferentes fontes, em espanhol e inglês, e verificar paralelos entre os critérios de relevância aplicados por agências de notícia e veículos jornalísticos e as generalizações propostas por Saracevic (2009) sobre como usuários de sistemas de informação inferem relevância, a análiseé apresentada em quatro partes:

Na primeira (4.1), são mapeados os termos usados em inglês pelas agências AFP, AP e Reuters, e comparados com os resultados encontrados em pesquisa anterior (FERNANDEZ JÚNIOR, 2013) em textos das mesmas agências em espanhol, sendoos Termos Políticos reunidos numa matriz de Classificação Política nos dois idiomas. 
Na segunda parte (4.2), são mapeados os termos usados em textos em inglês em sites das emissoras de TV BBC e CNN e do jornal The New York Times, e comparados com os resultados encontrados nas três agências no mesmo idioma. Na terceira(4.3), são mapeados os termos em textos em espanhol das mesmas emissoras de TV e do jornal El País, e relacionados com os resultados dos veículos em inglês e das agências em espanhol.

Ao final (4.4), são comparados os resultados em todas as fontes pesquisadas em cada idioma e identificadas diferenças e semelhanças entre elas.

\subsection{Classificação Política em textos de agências de notícia em espanhol e inglês}

A análise dos serviços de três agências de notícia (AFP, AP e Reuters) revela termos usados por todas em espanhol e inglês; por todas em apenas um idioma, ou isoladamente por uma delas em um ou outro serviço. São notáveis algumas semelhanças entre agências em apenas um idioma, como a presença unânime de marxista somente em inglês e a ocorrência de social democrata restrita ao espanhol (Tabela 1). Tais coincidências, todavia, não parecem explicáveis pela troca de idioma, já que mesmo os termos usados exclusivamente em um deles teriam traduções ou correspondentes possíveis no outro.

Tabela 1: Termos de Classificação Política usados por agências em espanhol e inglês

\begin{tabular}{l|ccc|ccc}
\hline \multirow{2}{*}{$\begin{array}{l}\text { Cgências -Espanhol e Inglês } \\
\text { Classificação Política 2010 (\%TF) }\end{array}$} & \multicolumn{3}{|c|}{ ESPANHOL } & \multicolumn{3}{c}{ INGLÊS } \\
\cline { 2 - 7 } minoritário & AFP-e & AP-e & RTS-e & AFP-i & AP-i & RTS-i \\
radical & - & $1 \%$ & - & - & - & - \\
marxist & $1 \%$ & - & - & - & - & - \\
socialist & - & - & - & $4 \%$ & $7 \%$ & $1 \%$ \\
izquierda/ista /left-wing/ leftist & - & - & - & - & - & $0 \% *$ \\
oficialista / ruling & $1 \%$ & $2 \%$ & $2 \%$ & - & - & $6 \%$ \\
gobernante/ governing & $14 \%$ & $13 \%$ & $\mathbf{1 8} \%$ & $\mathbf{9} \%$ & $\mathbf{6 \%}$ & $\mathbf{1 3 \%}$ \\
center-left & $1 \%$ & - & $2 \%$ & - & $1 \%$ & - \\
ambientalista & - & - & - & - & - & $1 \%$ \\
verde & $4 \%$ & - & - & - & - & - \\
& $0 \% *$ & - & - & - & - & -
\end{tabular}




\begin{tabular}{|c|c|c|c|c|c|c|}
\hline ecologista & $1 \%$ & - & - & - & - & - \\
\hline opositor /opposition & $9 \%$ & $8 \%$ & $10 \%$ & $5 \%$ & $3 \%$ & $10 \%$ \\
\hline socialdemócrata & $11 \%$ & $1 \% *$ & - & - & - & - \\
\hline centro/centrista/ centrist & - & $1 \% *$ & $3 \%$ & $1 \%$ & $9 \%$ & $3 \%$ \\
\hline conservador & - & - & $1 \% *$ & - & - & - \\
\hline Total Termos & 9 & 6 & 6 & 4 & 5 & 7 \\
\hline
\end{tabular}

Leg.: \%TF = Termo entre Termos Fonte (* uma ocorrência). Em negrito, Termos comuns a todas as agências.

Os resultados apontam que, se "diferentes usuários usam critérios similares" para inferir relevância, "mas podem aplicar diferentes pesos" (SARACEVIC, 2009, p.128), agências de notícia diferentes usam critérios similares em um mesmo idioma para atribuir relevância a informações associadas aos candidatos, mas aplicam diferentes pesos.

De modo semelhante, serviços de uma mesma agência em idiomas diferentes usam critérios similares, mas podem aplicar diferentes pesos em cada um.

E assim como "diferentes escalas de relevância usam critérios similares, mas podem aplicar diferentes pesos" (idem, p.129),diferentes classificações políticas usam termos similares,mas podem aplicar diferentes pesos em um mesmo idioma e em idiomas diferentes.

A partir da questão levantada por Saracevic (ibidem, p.138) sobre se "os julgamentos de relevância são estáveis enquanto tarefas e outros aspectos mudam", este estudo sugere que, apesar de utilizar critérios similares, a classificação política de uma mesma agência varia quando muda o idioma, apresentando termos diferentes em cada idioma, e termos similares com diferentes pesos em cada um.

Mesmo assim, em paralelo com a pergunta sobre se "os julgamentos de relevância são consistentes entre julgadores ou um grupo de julgadores" (ibidem), pode-se apontar que há consistência entre a classificação política de serviços de uma mesma agência em idiomas diferentes, assim como de diferentes agências em um mesmo idioma, como indica a ocorrência da dicotomia oficialista/ruling $\mathrm{x}$ opositor/opposition em todos os serviços das agências, mesmo quando não predominante.

\subsection{Classificação Política em textos de veículos jornalísticos em inglês}

A análise de notícias em inglês coletadas em sites de veículos jornalísticos tradicionais em outras mídias - as emissoras de TV BBC e 
CNN e o jornal The New York Times (NYT) - corrobora os paralelos com as generalizações sugeridas por Saracevic: assim como usuários de sistemas de informação ao inferirem relevância, os veículos pesquisados também aplicam múltiplos critérios de relevância às informações associadas aos candidatos a presidente na eleição de 2010, e eles são usados interativamente.

Ainda que envolva uma quantidade menor de textos, a análise sugere que veículos diferentes, no mesmo idioma, usam critérios similares aos das agências de notícia transnacionais para atribuir relevância, através de uma tipologia comum de termos usados na representação dos candidatos.Mesmo com ordens de prioridade distintas, há predomínio dos Termos Funcionais nos dois veículos americanos (CNN, NYT), assim como nas agências AP e AFP. Mas enquanto a emissora prioriza o termo guerrilla ao se referir a Dilma Rousseff, o jornal o faz apenas uma vez, associando-a prioritariamente a chief of staff.

Apesar de privilegiar Tipos de Termo diferentes, a rede de TV BBC (Termos Partidários) apresenta mais pontos em comum com a agência Reuters (Termos Políticos) do que em relação aos outros veículos em inglês. Ambas de origem britânica, BBC e Reuters são as fontes com mais textos em seus respectivos grupos; registram o maior percentual de Termos Recorrentes (que aparecem mais de uma vez) entre todas as fontes pesquisadas no idioma, e adotam Termos Políticos semelhantes em relação aos principais candidatos.

As coincidências entre veículo e agência de origem cultural comum são ainda maiores entre as americanas CNN e AP, que adotam a mesma ordem de prioridade de Tipos de Termo, mas com pesos diferentes; são as únicas com predominância da dicotomia marxist x centrist, e as que mais utilizam os termos guerrilla e marxist em relação a Dilma Rousseff. Ambas têm as maiores médias de Ocorrência de Termo por Texto em seus grupos, com quase a mesma média de Termos Recorrentes, o que pode refletir o fato de a emissora ser cliente da agência.

Diferentemente do registrado entre as agências, nenhum Termo Político em inglês é comum a todos os veículos, que não recorrem a termos distintos dos usados pelas agências e utilizam um número menor de termos deste tipo. Entre os veículos, há mais termos comuns entre os americanos CNN e NYT, que utilizam left-wing, leftist e marxist em relação a Dilma, com pesos diferentes, enquanto apenas a BBC usa opposition em referência a José Serra, identificado como centrist pela emissora americana e sem ser associado a qualquer Termo Político pelo jornal (Tabela 2). 
Tabela 2: Termos de Classificação Política usados por agências e veículos em inglês

\begin{tabular}{|c|c|c|c|c|c|c|}
\hline \multirow{2}{*}{$\begin{array}{l}\text { Agências e Veículos - Inglês } \\
\text { Classificação Política 2010(\%TF) }\end{array}$} & \multicolumn{3}{|c|}{ AGÊNCIAS } & \multicolumn{3}{|c|}{ VEÍCULOS } \\
\hline & RTS-i & AP-i & AFP-i & BBC-i & $\mathrm{CNN}-\mathrm{i}$ & NYT \\
\hline Marxista & $1 \%$ & $7 \%$ & $4 \%$ & - & $8 \%$ & $2 \% *$ \\
\hline Socialista & $0 \% *$ & - & - & - & - & - \\
\hline left-wing/leftist & $6 \%$ & - & - & - & $8 \%$ & $5 \%$ \\
\hline Ruling & $13 \%$ & $6 \%$ & $9 \%$ & $2 \%$ & $3 \% *$ & - \\
\hline governing & - & $1 \%$ & - & $8 \%$ & - & $2 \% *$ \\
\hline center-left & $1 \%$ & - & - & $2 \%$ & - & - \\
\hline opposition & $10 \%$ & $3 \%$ & $5 \%$ & $3 \%$ & - & - \\
\hline Centrista & $3 \%$ & $9 \%$ & $1 \%$ & - & $8 \%$ & - \\
\hline Total Termos & 7 & 5 & 4 & 4 & 4 & 3 \\
\hline
\end{tabular}

Leg.: \%TF = Termo entre Termos Fonte (* uma ocorrência).

\subsection{Classificação Política em textos de veículos jornalísticos em espanhol}

A análise de textos de três veículos em espanhol ratifica os paralelos com as generalizações sugeridas por Saracevic (2009) sobre como usuários de sistemas de informação inferem relevância, e revela semelhanças com os critérios adotados por agências de notícia transnacionais para atribuir relevância, com base em uma tipologia comum de termos, aplicada na representação dos candidatos a presidente do Brasil na eleição de 2010.

A comparação de textos das emissoras BBC e CNN em espanhol e inglês demonstra que, como nas agências, classificações políticas de um mesmo veículo em idiomas diferentes usam termos diferentes em cada idioma, e termos semelhantes com diferentes pesos em cada um.

Da mesma forma, alguns termos são usados por veículos em apenas um idioma, mas enquanto em inglês não há nenhum Termo Político comum a todos os veículos, em espanhol há unanimidade no uso de oficialista, gobernante e opositor, com pesos diferentes. Também como as agências, os veículos usam mais termos em espanhol (10) do que em inglês (7), mas, ao contrário delas - onde oficialista/ruling e opositor/opposition estão presentes em todas - nenhum termo é comum a todos os veículos nos dois idiomas (Tabela 3 ). 
Tabela 3: Termos de Classificação Política usados por veículos em espanhol e inglês

\begin{tabular}{|c|c|c|c|c|c|c|}
\hline \multirow{2}{*}{$\begin{array}{l}\text { Veículos - Espanhol e Inglês } \\
\text { Classificação Política } 2010 \text { (\%TF) }\end{array}$} & \multicolumn{3}{|c|}{ ESPANHOL } & \multicolumn{3}{|c|}{ INGLÊS } \\
\hline & BBC-e & CNN-e & El País & BBC-i & CNN-i & NYT \\
\hline marxista[leninista] /marxista & - & - & $1 \% *$ & - & $8 \%$ & $2 \% *$ \\
\hline Comunista & - & - & $1 \% *$ & - & - & - \\
\hline izquierdista / left-wing/leftist & - & $2 \% *$ & - & - & $8 \%$ & $5 \%$ \\
\hline oficialista / ruling & $16 \%$ & $10 \%$ & $4 \%$ & $2 \%$ & $3 \% *$ & - \\
\hline gobernante / governing & $3 \%$ & $4 \%$ & $1 \% *$ & $8 \%$ & - & $2 \% *$ \\
\hline Ambientalista & $1 \%$ & - & - & - & - & - \\
\hline Ecologista & - & - & $7 \%$ & - & - & - \\
\hline Verde & - & - & $1 \% *$ & - & - & - \\
\hline center-left & - & - & - & $2 \%$ & - & - \\
\hline opositor /opposition & $10 \%$ & $14 \%$ & $4 \%$ & $3 \%$ & - & - \\
\hline socialdemocrata & - & $2 \% *$ & $6 \%$ & - & - & - \\
\hline centrist & - & - & - & - & $8 \%$ & - \\
\hline Total Termos-conceitos & 4 & 5 & 8 & 4 & 4 & 3 \\
\hline
\end{tabular}

Leg.: \%TF = Termo entre Termos Fonte (* uma ocorrência). Em negrito, Termos comuns em um idioma.

Afora ocorrências isoladas de marxista e comunista(associados indiretamente a Dilma Rousseff) no El País, os veículos em espanhol, como em inglês, recorrem prioritariamente aos mesmos termos usados pelas agências, com oito termos-conceitos presentes nos dois grupos de fontes, dos 15 mapeados no idioma- quase o dobro dos oito adotados em inglês, sete deles comuns aos dois grupos.

Parte dessa diferença está na adoção de Termos Políticos ambientalista, ecologista, verde - associados a Marina Silva, apenas em espanhol, por quatro (AFP, EFE, BBC e El País) das sete fontes no idioma, uma opção aparentemente mais de cunho organizacional e cultural do que idiomático, já que termos correspondentes poderiam ser usados em inglês.

Apesar de a análise dos textos da BBC, como os da Reuters, sugerir estabilidade nos critérios de relevância nos dois idiomas, a comparação entre serviços da CNN em espanhol e inglês mostra que, como nas 
agências AP e AFP, os critérios de relevância de um mesmo veículo variam quando o idioma muda.

Mesmo assim, o predomínio dos Termos Partidários e Políticos como os dois tipos mais usados em todos os veículos em espanhol corrobora a ideia de que, como entre agências, há consistência entre os critérios de relevância de diferentes veículos e agências no mesmo idioma, apesar da variação de termos e pesos entre eles (Tabela 4).

Tabela 4: Termos de Classificação Política usados por agências e veículos em espanhol

\begin{tabular}{|c|c|c|c|c|c|c|c|}
\hline \multirow{2}{*}{$\begin{array}{l}\text { Agências e Veículos - Espanhol } \\
\text { Classificação Política } 2010 \text { (\%TF) }\end{array}$} & \multicolumn{4}{|c|}{ AGÊNCIAS } & \multicolumn{3}{|c|}{ VEÍCULOS } \\
\hline & AFP-e & AP-e & EFE & RTS-e & BBC-e & CNN-e & El País \\
\hline minoritário & - & $1 \%$ & - & - & - & - & - \\
\hline radical & $1 \%$ & - & - & - & - & - & - \\
\hline marxista[leninista] & - & - & - & - & - & - & $1 \% *$ \\
\hline Comunista & - & - & - & - & - & - & $1 \% *$ \\
\hline izquierda/ista / left-wing/ leftist & $1 \%$ & $2 \%$ & $1 \%$ & $2 \%$ & - & $2 \% *$ & - \\
\hline oficialista / ruling & $14 \%$ & $13 \%$ & $15 \%$ & $18 \%$ & $16 \%$ & $10 \%$ & $4 \%$ \\
\hline gobernante/governing & $1 \%$ & - & $1 \%$ & $2 \%$ & $3 \%$ & $4 \%$ & $1 \% *$ \\
\hline ambientalista & $4 \%$ & - & - & - & $1 \%$ & - & - \\
\hline ecologista & $1 \%$ & - & $1 \%$ & - & - & - & $7 \%$ \\
\hline Verde & $0 \% *$ & - & $1 \%$ & - & - & - & $1 \% *$ \\
\hline opositor /opposition & $9 \%$ & $8 \%$ & $18 \%$ & $10 \%$ & $10 \%$ & $14 \%$ & $4 \%$ \\
\hline socialdemócrata & $11 \%$ & $1 \% *$ & $1 \%$ & - & - & $2 \% *$ & $6 \%$ \\
\hline centro/centrista/ centrist & - & $1 \% *$ & $0 \% *$ & $3 \%$ & - & - & - \\
\hline Conservador & - & - & - & $1 \% *$ & - & - & - \\
\hline Derecha & - & - & $0 \% *$ & - & - & - & - \\
\hline Total Termos-conceitos & 9 & 6 & 9 & 6 & 4 & 5 & 8 \\
\hline
\end{tabular}

Leg.: \%TF $=$ Termo entre Termos Fonte ( $*$ uma ocorrência). Em negrito, Termos comuns a agências e veículos.

\subsection{Agências e veículos: semelhanças e diferenças}

Dos 13 serviços noticiosos analisados, apenas o da CNN em espanhol não utiliza todos os cinco Tipos de Termo mapeados, deixando de usar os Termos Relacionais - que representam $11 \%$ dos termos da 
emissora em inglês. Apesar da tipologia comum, agências e veículos aplicam pesos e ordens de prioridade diferentes entre si e entre idiomas, mas com semelhanças entre fontes no mesmo idioma, e variações similares entre eles - como o predomínio dos Termos Partidários nos três veículos e na AP em espanhol (Tabela 5), e dos Termos Funcionais em quatro das seis fontes em inglês (Tabela 6 ).

Tabela 5:Tipos de Termo usados por agências e veículos em espanhol

\begin{tabular}{l|rrrr|rrr}
\hline \multirow{2}{*}{$\begin{array}{l}\text { Agências e Veículos - } 2010 \\
\text { Tipos em Espanhol (\% TF) }\end{array}$} & \multicolumn{4}{|c|}{ AGÊNCIAS } & \multicolumn{3}{c}{ VEíCULOS } \\
\cline { 2 - 7 } & AFP-e & AP-e & EFE* & RTS-e & BBC-e & CNN-e & EI País \\
\hline PARTIDÁRIO & $7 \%(4)$ & $32 \%(1)$ & $38 \%(2)$ & $22 \%(3)$ & $36 \%(1)$ & $48 \%(1)$ & $27 \%(1)$ \\
POLÍTICO & $40 \%(1)$ & $25 \%(2)$ & $38 \%(1)$ & $36 \%(1)$ & $30 \%(2)$ & $22 \%(2)$ & $25 \%(2)$ \\
FUNCIONAL & $19 \%(3)$ & $19 \%(3)$ & $8 \%(4)$ & $23 \%(2)$ & $17 \%(3)$ & $8 \%(4)$ & $15 \%(4)$ \\
PESSOAL & $28 \%(2)$ & $15 \%(4)$ & $11 \%(3)$ & $10 \%(4)$ & $9 \%(4)$ & $22 \%(2)$ & $23 \%(3)$ \\
RELACIONAL & $6 \%(5)$ & $8 \%(5)$ & $5 \%(5)$ & $9 \%(5)$ & $7 \%(5)$ & $0 \%(5)$ & $9 \%(5)$ \\
\hline
\end{tabular}

Leg: TF = Total Termos Fonte(ordem de prioridade). ${ }^{*}$ Empate percentual resulta de arredondamento

Tabela 6: Tipos de Termo usados por agências e veículos em inglês

\begin{tabular}{l|ccc|ccr}
\hline \multirow{2}{*}{$\begin{array}{l}\text { Agências e Veículos - 2010 } \\
\text { Tipos em Inglês (\% TF) }\end{array}$} & \multicolumn{3}{|c|}{ AGÊNCIAS } & \multicolumn{3}{c}{ VEícULOS } \\
\cline { 2 - 7 } & AFP-i & AP-i & RTS-i & BBC-i & CNN-i & \multicolumn{1}{c}{ NYT } \\
\hline FUNCIONAL & $40 \%(1)$ & $26 \%(1)$ & $26 \%(2)$ & $14 \%(4)$ & $49 \%(1)$ & $37 \%(1)$ \\
POLÍTICO & $19 \%(3)$ & $25 \%(2)$ & $34 \%(1)$ & $15 \%(3)$ & $27 \%(2)$ & $9 \%(5)$ \\
PESSOAL & $21 \%(2)$ & $23 \%(3)$ & $14 \%(4)$ & $25 \%(2)$ & $11 \%(3)$ & $26 \%(2)$ \\
RELACIONAL & $18 \%(4)$ & $15 \%(4)$ & $11 \%(5)$ & $12 \%(5)$ & $11 \%(3)$ & $19 \%(3)$ \\
PARTIDÁRIO & $3 \%(5)$ & $11 \%(5)$ & $15 \%(3)$ & $34 \%(1)$ & $3 \%(5)$ & $16 \%(4)$ \\
\hline
\end{tabular}

Leg: TF = Total Termos Fonte (ordem de prioridade).

Ainda assim, é possível apontar alguma estabilidade de critérios também entre fontes que alteram a ordem de prioridade dos Tipos de Termo entre idiomas, como o mesmo peso (25\%) dos Termos Políticos em textos da AP em espanhol e inglês - o segundo mais usado em ambos - e o percentual igual $(40 \%)$ do tipo mais usado pela AFP nos dois idiomas apesar de trocar os Termos Políticos, predominantes em espanhol, pelos Funcionais em inglês.

Mesmo com ordens de prioridade distintas entre agências e veículos, há mais coincidências entre as fontes em espanhol do que em inglês, com quatro delas priorizando os Termos Partidários e três os Políticos - o 
segundo tipo mais usado nas demais - e todas tendo os Termos Relacionais como o tipo menos adotado.

As semelhanças e particularidades entre fontes, e diferenças entre idiomas, ficam mais nítidas na análise dos Termos Políticos utilizados por cada uma. Mais uma vez, há coincidências entre agências e veículos no mesmo idioma - como o uso unânime de oficialista e opositor em espanhol, e as presenças de marxist em cinco das seis fontes em inglês (e apenas uma em espanhol) e de socialdemócrata em cinco das sete em espanhol (e nenhuma em inglês) - sugerindo que há consistência entre os critérios de relevância de agências e veículos em idiomas diferentes.

Considerando que todos os veículos são potenciais clientes das agências pesquisadas, os resultados desta análise sugerem que fatores organizacionais e culturais, mais do que linguísticos, contribuem para aproximar os critérios de relevância aplicados e as classificações políticas adotadas em um mesmo idioma por veículos jornalísticos tradicionais em outras mídias e agências de notícia transnacionais,na representação dos candidatos a presidente do Brasil na eleição de 2010.

\section{Conclusão}

A análise de textos sobre a eleição presidencial brasileira de 2010, distribuídos na internet por quatro agências de notícias transnacionais (AFP, AP, EFE e Reuters) e quatro veículos jornalísticos tradicionais em outras mídias (os jornais El País e The New York Times e as emissoras de TV BBC e CNN), mostra que os dois grupos de fontes de informação utilizaram uma tipologia comum de termos na representação dos candidatos Dilma Rousseff (PT), José Serra (PSDB), Marina Silva (PV) e Plínio de Arruda Sampaio (PSOL). Além de adotarem os mesmos Tipos de Termo, agências e veículos recorreram a classificações políticas semelhantes em espanhol e em inglês, com diferenças entre idiomas e coincidências entre fontes de origem cultural comum.

Os resultados corroboram paralelos com as generalizações de Saracevic (2009) sobre como usuários de sistemas de informação inferem relevância, ao sugerir que agências e veículos diferentes, no mesmo idioma,usam critérios similares para atribuir relevância às informações associadas a cada candidato, aplicando diferentes pesos e múltiplos critérios, usados interativamente, a partir de uma tipologia comum de termos adotada com variações em espanhol e inglês.

Dentre as cinco fontes analisadas nos dois idiomas, apenas Reuters e BBC priorizam o mesmo tipo de Termo em ambos - Políticos e Partidários, respectivamente - mas somente a Reuters repete a ordem de prioridade dos demais. AFP, AP e CNN recorrem a tipos e ordens de 
prioridade diferentes em inglês e espanhol, indicando que os critérios de relevância entre agências e veículos variam quando o idioma muda. Uma demonstração disso é a inversão de prioridade dos Termos Partidários nas americanas AP e CNN, onde são o tipo mais usado em espanhol e o menos presente em inglês. Mesmo entre as fontes que apresentam critérios semelhantes nos dois idiomas, há variações de peso no uso de termos similares em cada um.

A análise dos textos de veículos jornalísticos reforça o paralelo de que diferentes classificações políticas [no mesmo idioma] usam termos similares, mas aplicam diferentes pesos, como identificado entre agências. Já a comparação entre fontes com textos em espanhol e inglês indica que diferentes classificações políticas usam termos diferentes em cada idioma, e termos similares com diferentes pesos, refletindo matrizes de classificação distintas entre fontes no mesmo idioma, e entre idiomas na mesma fonte.

Os resultados revelam coincidências entre agências e veículos de origem cultural comum, como a adoção da dicotomia marxist $\mathrm{x}$ centrist apenas nas americanas AP e CNN - que têm ordem de prioridade de tipos similar nos dois idiomas -, e a ausência do termo social demócrata nas "britânicas" Reuters e BBC. É destacável também que socialdemócrata ocorra somente em espanhol e apenas uma vez nas americanas AP e CNN, tendo mais peso nas fontes de origem latina, com predomínio nas referências a José Serra na AFP e no El País.

Deste modo, apesar das diferenças de ordem organizacional e idiomática, os resultados deste estudo sugerem que há consistência entre as classificações políticas de agências de notícia e veículos jornalísticos no mesmo idioma, e de fontes de origem cultural comum em idiomas diferentes.

Se por um lado a variação do peso dado ao mesmo termo em diferentes fontes de informação reflete critérios organizacionais de cada uma, a coincidência no uso de determinados termos apenas em espanhol ou inglês não parece explicável somente pela mudança de idioma. Nessas variações, há mais coincidências entre fontes de origem cultural comum, mesmo em idiomas diferentes, sugerindo que os fatores organizacionais, idiomáticos e culturais atuam conjuntamente na definição das matrizes de classificação política de agências de notícias transnacionais e veículos jornalísticos tradicionais, utilizadas na representação dos candidatos a presidente do Brasil na eleição de 2010.

A comparação dos resultados aqui apresentados com a análise de material semelhante em eleições subsequentes, além de permitir a verificação dos paralelos propostos,poderá avaliar se, assim como "diferentes seleções são feitas em diferentes estágios usando critérios 
similares, mas possivelmente com diferentes pesos" (SARACEVIC, 2009, p. 129, grifo do autor), diferentes seleções de termos são feitas por agências e veículos em diferentes eleições usando critérios similares, mas possivelmente com diferentes pesos; e se, assim como "o que é tópico muda com o progresso no tempo" (idem), o que é termo político muda entre eleições.

Ao focar nos critérios de relevância e nas classificações políticas presentes no discurso jornalístico em sites de notícias internacionais, procuramos aprofundar o entendimento das representações encontradas em textos armazenados em bases de dados digitais, numa época em que a internet exerce papel central na distribuição de informação em escala global, contribuindo para a confluência de pesquisas comuns aos campos da Ciência da Informação e da Comunicação.

\section{Referências}

ALDÉ, A.; VEIGA, L.F. Recepção da comunicação política.In: RUBIM, A.A.C. (Org.) Comunicação e política:conceitos e abordagens. Salvador: Edufba, p.483-513, 2004.

BIOLCHINI, J.C.A. Semântica e cognição em bases de conhecimento: do vocabulário controlado à ontologia. Data Grama Zero - Revista de Ciência da Informação, v. 2, n.5, out. 2001.

BOWKER, G.C.; STAR, S.L. Sortingthings out: classification and its consequences. Cambridge: MIT Press, 2000.

CAMARA JR., J.M. Dicionário de lingüística e gramática. Petrópolis: Vozes, 1985.

ENTMAN, R.M. Framing: toward clarification of a fractured paradigm. Journalof Communication, v.43, n.4, p.51-58, 1993.

FERNANDEZ JÚNIOR, F.E. Classificação política e discurso jornalístico: como agências de notícia transnacionais representam candidatos a presidente do Brasil nas eleições de 1989 e 2010.2013. 246 f. Tese (Doutorado em Ciência da Informação) - Escola de Comunicação, Universidade Federal do Rio de Janeiro, Rio de Janeiro, 2013.

GAMSON, W.A.; MODIGLIANI, A. Media discourse and public opinion on nuclear power: a constructionist approach. American Journal of Sociology, Chicago: The University of Chicago Press, v. 95, n. 1, p.1-37, Jul,1989.

GITLIN, T. The whole world is watching. Berkeley: University of California Press, 1980. 
HJØRLAND, B..The foundation of the concept of relevance.Journal of the American Society for Information Science and Technology, v.61, n. 2, p.217-237, 2010.

LAKOFF, G. Woman, fire and dangerous things: what categories reveal about mind. Chicago: University of Chicago Press, 1987.

LIMA, V.A. de.Cenários de representação da política, CR-P.In:RUBIM, A.A.C. (org.) Comunicação e política: conceitos e abordagens. Salvador: Edufba, p.9-40, 2004.

OLMSTEAD, K.; MITCHELL, A.; ROSENSTIEL, T. Navigating news online.Pew Research Center's Project for Excellence in Journalism, 9 maio 2011.Disponível em:

http://www.journalism.org/analysis report/navigating news online. Acesso em: 10 nov. 2015

ORRICO, E.G.D.; OLIVEIRA, C.I. de. A linguagem na construção do sentido: discurso e organização do conhecimento. In. GONZÁLEZ DE GÓMEZ, M.N.; ORRICO, E.G.D. (Org.) Políticas de memória e informação:reflexos na organização do conhecimento. Natal: Ed. UFRN, p.143-170, 2006.

PAREDES SILVA, V.L. Continuidade de referência: nomes, pronomes e anáfora zero emgêneros da fala e da escrita.Revista Lingüística, Rio de Janeiro, v.3. n.1, p.159-178, jun. 2007.

PATERSON, C. News agency dominance in international news on the internet. Centre for International Communications Research, Leeds University, 2006.

PINHEIRO, L.V.R..Informação, esse obscuro objeto da Ciência da Informação.MORPHEUS: Revista Eletrônica em Ciências Humanas Informação e Sociedade, Rio de Janeiro, UNIRIO, v.2, n.4, 2004.

SARACEVIC, T. Relevance: a review of the literature and a framework for the thinking on the notion in information science. In: BRAGA, G.M; PINHEIRO, L.V.R. (Org.). Desafios do impresso ao digital:questões contemporâneas de informação e conhecimento. Brasília: IBICT/UNESCO, p.15-168, 2009.

SCHUDSON, M. Discovering the news:a social history of American Newspapers. New York: Basic Books Inc., 1978.

TRAQUINA, N. O estudo do jornalismo no Século XX. São Leopoldo: Ed. Unisinos, 2001. 
TUCHMAN, G. Making news by doing work: routinizing the unexpected. The American Journal of Sociology, Chicago: The University of Chicago Press. v. 79, n. 1, p. 110-131, July 1973. Disponível em:

http://www.jstor.org/stable/2776714.Acesso em: 10 nov. 2015. 